\title{
LV geometric and substrate remodelling in patient with Ebstein anomaly - a deep insight from MRI T1 mapping fibrosis imaging
}

Dan Yang ${ }^{1 *}$, Jiayu Sun ${ }^{2}$, Ke Wan ${ }^{1}$, Yong Luo ${ }^{1}$, Hong Liu' ${ }^{1}$ Wei Cheng ${ }^{2}$, Tianjiang Zhang ${ }^{3}$, Yucheng Chen ${ }^{1}$

From 19th Annual SCMR Scientific Sessions

Los Angeles, CA, USA. 27-30 January 2016

\section{Background}

Ebstein anomaly is a kind of congenital defect of tricuspid valve mainly affecting the right ventricle, recent evidence have suggested the LV geometry remodeling in patient with Ebstein anomaly due to potential RV-LV coupling. The aim of this study-we investigated the interaction between abnormal right ventricle and left ventricle and whether myocardial fibrosis is the basis of abnormal left ventricle remodeling in ebstein abnomaly by using $\mathrm{T} 1$ mapping technique of cardiac magnetic resonance.

\section{Methods}

Twenty patients with ebstein's anomaly and twenty matched normal controls were prospectively included in this study. All subjects received a comprehensive cardiac MRI scanning according to a standard protocol, which consisted of pre- and post-contrast T1 mapping for calculating ECV to quantify myocardial fibrosis of left ventricle. LV geometry and function data. Bi-ventricular volume, function and severity of ebstein anomaly were also analyzed.

\section{Results}

In patients with Ebstein anomaly, LV volume was mildly decreased and EF also mildly reduced .ECV of LV myocardium in ebstein was larger than that of normal controls $(28.76 \pm 4.40$ vs $23.39 \pm 3.56 \mathrm{p}=0.003)$. ECV correlated well with severity index of RV malformation and LVEF in Ebstein's patients group. The native-T1 value of LV myocardium in ebstein's was longer than that of in normal controls with $\mathrm{p}=0.04$.

\section{Conclusions}

LV myocardial fibrosis and remodeling evidently existed in patients with ebstein's anomaly which was strongly correlated to the severity index of ebstein. Myocardial fibrosis plays a role in LV remodeling which directly results from LV deformity in ebstein' patients.

\section{Authors' details}

${ }^{1}$ Cardiology Division, West China Hospita Sichuan University, Chengdu, China. ${ }^{2}$ Radiology Department, West China Hospital, Sichuan University, Chengdu, China. ${ }^{3}$ Siemens MR Northeastern Collaboration, Beijing, China.

Published: 27 January 2016

doi:10.1186/1532-429X-18-S1-P159

Cite this article as: Yang et al:: LV geometric and substrate remodelling in patient with Ebstein anomaly - a deep insight from MRI T1 mapping fibrosis imaging. Journal of Cardiovascular Magnetic Resonance 2016 18(Suppl 1):P159. 


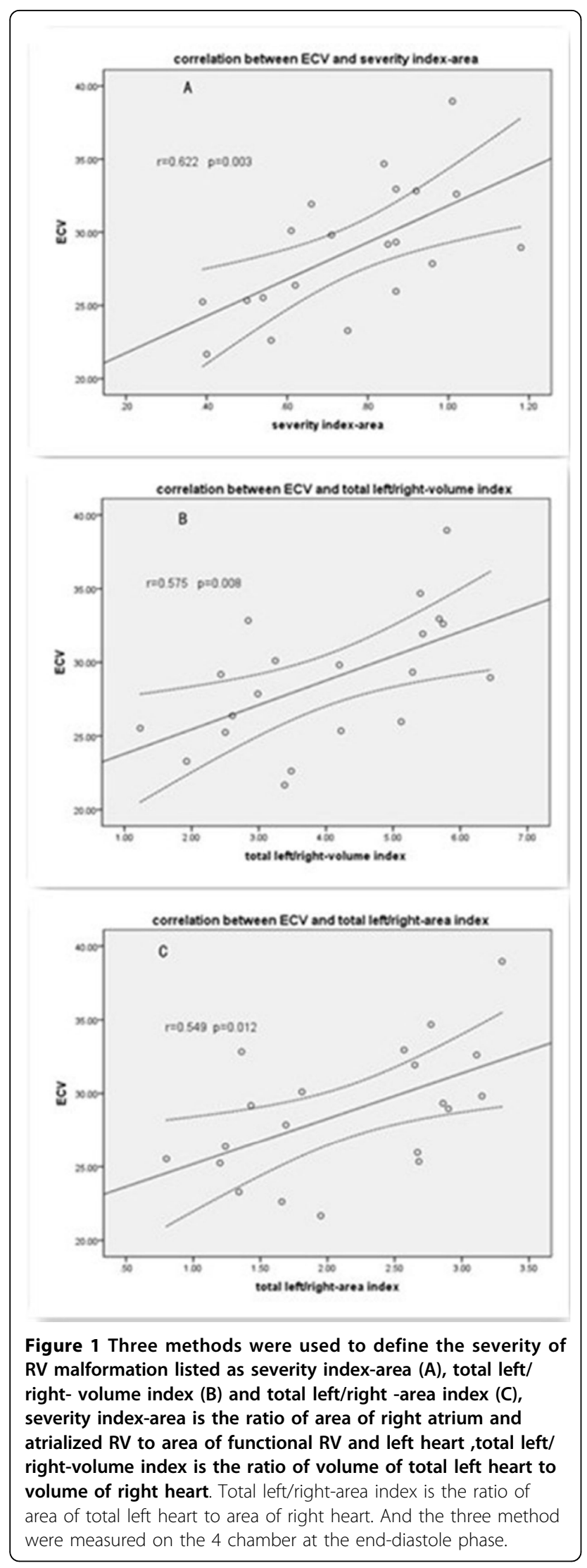

